\title{
Enhanced Raman scattering in thermally poled sodium-niobium borophosphate glasses
}

\author{
Marc Dussauze ${ }^{a)}$ \\ Theoretical and Physical Chemistry Institute, National Hellenic Research Foundation, 48 Vass. \\ Constantinou Avenue, 11635 Athens, Greece
}

Evelyne Fargin

Institut de Chimie de la Matière Condensée de Bordeaux, UPR 9048 CNRS, Chateau Brivazac, Avenue du Dr. Schweitzer, 33608 Pessac Cedex, France

Vincent Rodriguez

Institut des Sciences Moléculaires, UMR 5255 CNRS, Groupe de Spectroscopie Moléculaire, Université Bordeaux I, 351 cours de la Libération, 33405 Talence Cedex, France

\section{Artem Malakho}

Institut de Chimie de la Matière Condensée de Bordeaux, UPR 9048 CNRS, Chateau Brivazac, Avenue du Dr. Schweitzer, 33608 Pessac Cedex, France

\author{
Efstratios Kamitsos \\ Theoretical and Physical Chemistry Institute, National Hellenic Research Foundation, 48 Vass. \\ Constantinou Avenue, 11635 Athens, Greece
}

(Received 19 December 2006; accepted 6 March 2007; published online 30 April 2007)

\begin{abstract}
Micro-Raman analysis has been carried out on the cross section of thermally poled sodium-niobium borophosphate glasses. We were able to measure with accuracy an enhanced Raman response from the nonlinear optical (NLO) layer formed at the anode side of the poled glasses. The thickness of the NLO layer has been estimated. Several spectral changes were observed within this layer, which manifest important structural rearrangements after thermal poling. Possible mechanisms leading to structural reorganization in the space charge region are discussed. (C) 2007 American Institute of Physics. [DOI: 10.1063/1.2724798]
\end{abstract}

\section{INTRODUCTION}

The development of photonics applications, e.g., as electro-optic switches and modulators, provide a means to implement low-cost active optical devices with low losses and good fiber compatibility. In this context, recent works have demonstrated that large values of second order nonlinear optical coefficient $\left[\chi^{(2)}\right]$ can be obtained in thermally poled inorganic glasses or transparent glass ceramics. ${ }^{1-3} \mathrm{Be}-$ cause of the good thermal, optical, and mechanical stabilities of such materials, they can be considered as potential candidates in this domain.

In previous works, we have reported that large $\chi^{(2)}$ values can be reproducibly induced in niobium sodiumborophosphate glasses in bulk or thin film forms by thermal poling treatment. ${ }^{4,5}$ In such glasses, the second order nonlinear optical (NLO) response has been correlated to a third order NLO process through interaction of a high $\chi^{(3)}$ value, linked to the presence of a three-dimensional (3D) niobate network, ${ }^{6-8}$ with a static internal electric field $\left(E_{\text {int }}\right)$ induced by a migration of sodium ions away from the anode surface. $^{4,9}$

$$
\chi^{(2)}=3 \chi^{(3)} E_{\text {int }} \text {. }
$$

Nevertheless, the mechanisms responsible for the formation of the space charge region at the anode $\left(E_{\text {int }}\right)$ are still not

\footnotetext{
${ }^{a)}$ Author to whom correspondence should be addressed FAX: $(+30) 210$ 7273794; electronic mail: mdussauze@eie.gr
}

understood. Notably, the extent of displacement of the positive charge (sodium ions) in the NLO layer would induce alone an internal field $10^{4}$ times larger than that corresponding to the measured $\chi^{(2)}$ value. ${ }^{4}$ Thus, structural rearrangements should prevent the development of such a strong internal field. This work is an attempt to reveal possible reorganizations of the glass matrix by employing microRaman spectroscopy.

\section{EXPERIMENT}

Bulk glasses of composition $(1-x)\left(0.95 \mathrm{NaPO}_{3}\right.$ $\left.+0.05 \mathrm{Na}_{2} \mathrm{~B}_{4} \mathrm{O}_{7}\right)+x \mathrm{Nb}_{2} \mathrm{O}_{5}$, noted by bpn $x$ where $x$ is the mole fraction of $\mathrm{Nb}_{2} \mathrm{O}_{5}, x=0.35,0.4$, and 0.45 , were elaborated by a classical solid phase process. High purity reagent powders of $\mathrm{NaPO}_{3}, \mathrm{Na}_{2} \mathrm{~B}_{4} \mathrm{O}_{7}$, and $\mathrm{Nb}_{2} \mathrm{O}_{5}$ were mixed, grounded, and melted in platinum crucibles at a temperature of $1300{ }^{\circ} \mathrm{C}$. Glass samples were obtained by quenching the melt in a brass preform (1 mm depth). The glass was annealed in air at $10^{\circ} \mathrm{C}$ below glass transition temperature, and then cut and polished on both sides to give transparent specimens $1 \mathrm{~mm}$ thick.

Thermal poling was done in air at ambient pressure and at a temperature of $230{ }^{\circ} \mathrm{C}$, using a silicon wafer at the anode and a soda lime glass plate at the cathode to prevent reduction of the sample. The glass plates were heated at the poling temperature for at least one hour before applying an 


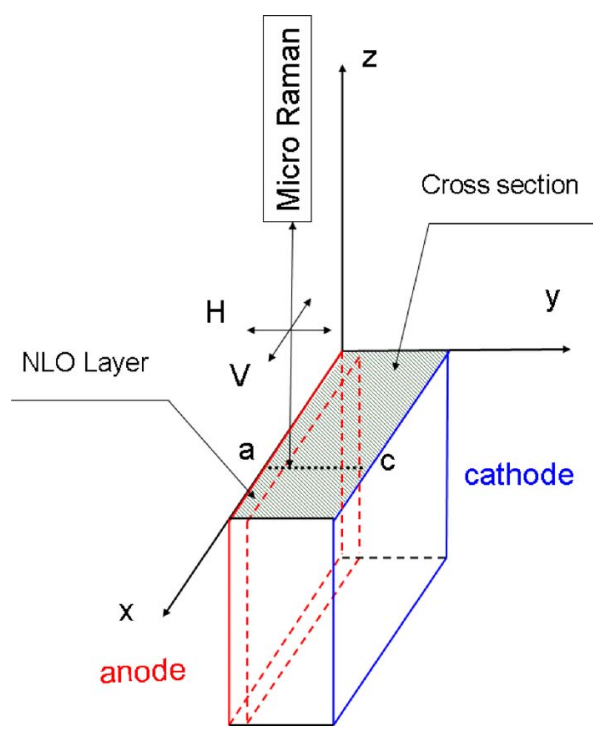

FIG. 1. (Color online) Schematic representation of the experimental configuration. The $X-Y$ face shows the cross section of a poled glass specimen studied by micro-Raman spectroscopy in the backscattering geometry. $\mathrm{V}$ and $\mathrm{H}$ indicate the polarization of the exciting laser beam.

electric field of $2 \mathrm{kV}$. After a thermal poling treatment of $30 \mathrm{~min}$ the samples were cooled down to room temperature before removing the dc bias.

Raman spectra were recorded at room temperature on a Labram confocal micro-Raman instrument (Horiba/JobinYvon) in the backscattering geometry, using the $632.8 \mathrm{~nm}$ excitation line of a $\mathrm{He} / \mathrm{Ne}$ laser and typical resolution of $3 \mathrm{~cm}^{-1}$. The spectrophotometer includes a holographic Notch filter for Rayleigh rejection, a microscope equipped with a $100 \times$ objective, and a Charge Coupled Device (CCD) air cooled detector.

Each poled glass plate was cut with the cross section parallel to the applied dc field ( $X-Y$ plane in Fig. 1), and was slightly polished to obtain uniform flat surfaces. Raman spectra were collected from the cross section in steps of $0.5 \mu \mathrm{m}$ from the anode to the cathode region, e.g., along line $a-c$ in Fig. 1, with a $\sim 1.5 \mu \mathrm{m}$ spatial resolution. Spectra were recorded with the incident laser beam polarized either along or perpendicular to the direction of the applied field for poling, i.e., $\mathrm{H}$ and $\mathrm{V}$ in Fig. 1, respectively. Laser beam focus and intensity were kept constant during spectral acquisition at different points of the cross section.

\section{RESULTS}

Raman spectra were measured along the cross section of three poled glasses with compositions bpn35, bpn40, and bpn45. For each glass composition studied, the spectra representing the bulk (middle of line $a-c$ in Fig. 1) and the cathode side (close to point $\mathbf{c}$ in Fig. 1) were found to be identical. However, Raman spectra recorded at the anode region (close to point $\mathbf{a}$ in Fig. 1) revealed systematic changes within the first five microns from the anode surface (point a). Such progressive spectral variations are clearly observed in Fig. 2, where the whole Raman intensity recorded close to the anode appears twice as strong as that of the bulk glass response. In addition, outside the NLO layer, e.g., between 4

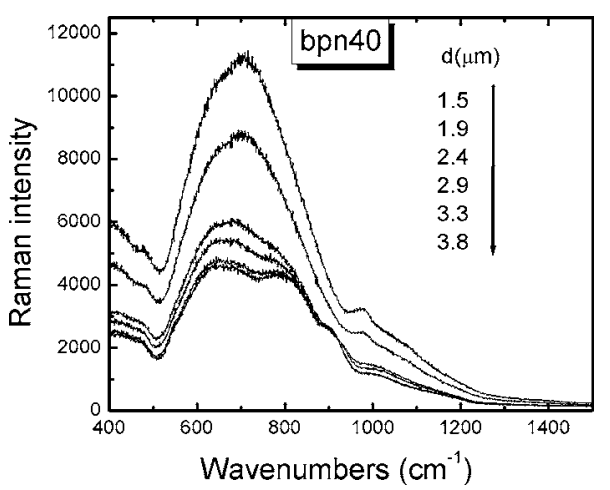

FIG. 2. (Color online) Micro-Raman spectra collected from the cross section at distances, $d$, from the anode surface of a poled glass with composition bpn40.

and $5 \mu \mathrm{m}$ from the anode side to the cathode side, the Raman spectra remain unchanged. Besides this overall spectral intensity modification, the shape of the Raman spectrum does vary significantly inside the NLO layer (Fig. 2).

Polarized Raman spectra $(\mathrm{VV}+\mathrm{VH}$ and $\mathrm{HV}+\mathrm{HH})$ of the bpn35 poled sample, measured inside the NLO layer, are reported in Fig. 3. No spectral modification is evidenced whatever the polarization of the incident beam is. Similar results were found for the other poled glasses studied in this work.

We focus now on the Raman band shape differences between the NLO layer and the bulk part of poled glasses. Figure 4 reports normalized Raman spectra of the three studied glasses collected at the anode side (NLO layer) and in the bulk. Regarding the phosphate entities in glass, on the first hand the symmetric stretching mode of the phosphate tetrahedral is expected in the range of $950-1200 \mathrm{~cm}^{-1}$ depending on their level of structural modification. ${ }^{6,10}$ On the other hand, a less intense symmetric stretching mode of P-O-P bridges is expected from around 650 to $750 \mathrm{~cm}^{-1}$. While the former band appears as a weak shoulder at around $1030 \mathrm{~cm}^{-1}$ in the Raman envelope of the bulk bpn35 glass, the latter one is probably hidden by a much stronger niobate band with a large envelope from 500 to $950 \mathrm{~cm}^{-1}$ attributed mainly to the symmetric stretching vibration of $\mathrm{Nb}-\mathrm{O}$ bonding moities. ${ }^{7,11}$ In particular, the higher frequency band or shoulder at $900 \mathrm{~cm}^{-1}$ is attributed to $\mathrm{NbO}_{6}$ octahedra with nonbridging oxygen atoms, $\mathrm{Nb}-\mathrm{O}^{-} \cdots \mathrm{Na}^{+}$, while modes associated with bridging bonds of the phosphoniobate network

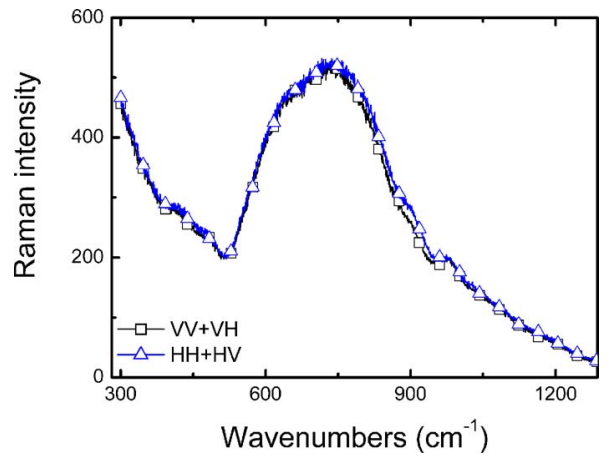

FIG. 3. (Color online) Micro-Raman spectra measured within the NLO layer with two polarization configurations. 


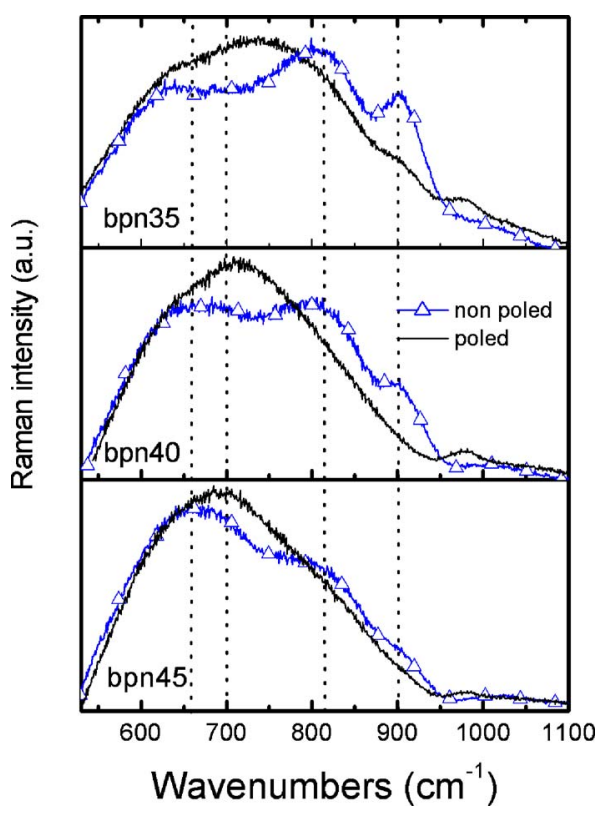

FIG. 4. (Color online) Micro-Raman spectra of three poled glass compositions recorded in the NLO layer and in the bulk.

are expected in the $500-850 \mathrm{~cm}^{-1}$ range. These include $\mathrm{NbO}_{6}$ octahedra bridging with niobium and phosphorus centers $\left(750-850 \mathrm{~cm}^{-1}\right)$ and $\mathrm{Nb}-\mathrm{O}-\mathrm{Nb}$ bonds in a $3 \mathrm{D}$ niobate network $\left(600-750 \mathrm{~cm}^{-1}\right)$. These two contributions are clearly discerned on the Raman spectra measured in the bulk (Fig. 4, unpoled area) and in addition, the lower frequency band gains relative intensity upon increasing the niobium oxide content. This is consistent with a gradual build up of a 3D niobate network from bpn35 to bpn45. Upon thermal poling, we observe in the NLO layer (anode side) only one strong band peaking at an intermediate frequency, e.g., $720 \mathrm{~cm}^{-1}$ for poled bpn40 and $690 \mathrm{~cm}^{-1}$ for poled bpn 45

In order to highlight further spectral differences induced by poling, Fig. 5 details Raman difference spectra between normalized spectra measured in the NLO layer and in the bulk of glasses bpn35 to bpn45. This figure shows a clear increase at $725 \mathrm{~cm}^{-1}$ and a decrease at 820 and $900 \mathrm{~cm}^{-1}$. Noticeably, the relative changes in the Raman spectra upon poling are more pronounced for glasses of lower niobium oxide content (bpn35).

\section{DISCUSSION}

\section{A. Raman intensity enhancement in the nonlinear optical layer}

A key result of this work concerns the effective probing of the NLO layer by micro-Raman spectroscopy. Figure 6 presents the integrated Raman intensity of the poled bpn40 glass from 400 to $1400 \mathrm{~cm}^{-1}$ versus distance from the anode surface that manifests a clear enhancement of the Raman signal in the NLO layer. According to the axial spatial resolution of this experiment $(\sim 1.5 \mu \mathrm{m})$, we estimate a thickness of the NLO layer in the range of 3-4 $\mu \mathrm{m}$. This result is in very good agreement with previous findings from Maker fringes analyses ${ }^{6}$ and depth profile determinations of sodium concentration ${ }^{4}$ on similar poled glasses.

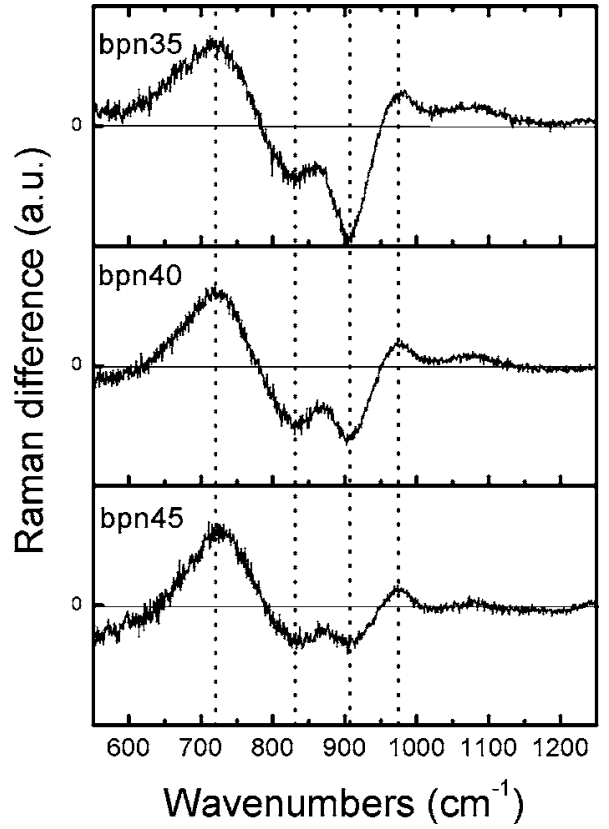

FIG. 5. (Color online) Micro-Raman difference spectra of three poled glass compositions, obtained by subtracting the normalized spectra collected in the NLO layer and in the bulk of each glass.

The origin of Raman intensity enhancement in the NLO layer is not known. If we consider only the interaction between light and material, the induced polarization, $P$, can be expanded in terms of the incident electric field $E_{\omega}$,

$$
P=\varepsilon_{0}\left[\chi^{(1)} E_{\omega}+\chi^{(2)} E_{\omega} E_{\omega}+\chi^{(3)} E_{\omega} E_{\omega} E_{\omega}+\ldots\right] .
$$

In terms of the internal static electric field, $E_{\text {int }}$, induced at the anode side, the induced polarization reads

$$
P=\varepsilon_{0}\left[\chi^{(1)}+2 \chi^{(2)} E_{\mathrm{int}}+3 \chi^{(3)} E_{\mathrm{int}} E_{\mathrm{int}}+\ldots\right] E_{\omega} .
$$

Typical orders of magnitude for susceptibilities are as follows: $\quad \chi^{(1)} \sim 10^{-2} \chi^{(2)} \sim 10^{-12} \mathrm{~m} / \mathrm{V} ; \quad \chi^{(3)} \sim 10^{-22} \mathrm{~m}^{2} / \mathrm{V}^{2}$. Based on such values and considering a static electric field of $E_{\text {int }} \sim 10^{9} \mathrm{~V} / \mathrm{m}$, the linear $\left[2 \chi^{(2)} E_{\text {int }}\right]$ and quadratic $\left[3 \chi^{(3)} E_{\text {int }} E_{\text {int }}\right]$ electro-optic terms should contribute to the total induced polarization $P$ at a level of $2 \times 10 \%$ and 3 $\times 1 \%$, respectively.

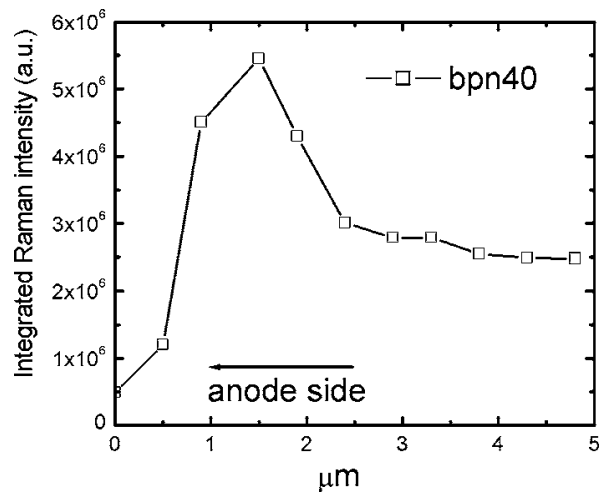

FIG. 6. (Color online) Integrated Raman intensity (ranging from 400 to $1400 \mathrm{~cm}^{-1}$ ) in steps of $0.5 \mu \mathrm{m}$ from the anode side of the bpn 40 poled glass. 
Focusing on the Raman response, we consider now the modulation of the induced polarization, given by Eq. (3), under coordinate $Q$ of a normal mode,

$$
\left(\frac{\partial P}{\partial Q}\right)_{0}=\varepsilon_{0} \frac{\partial}{\partial Q}\left[\left(\chi^{(1)}+2 \chi^{(2)} E_{\mathrm{int}}+3 \chi^{(3)} E_{\mathrm{int}}^{2}+\ldots\right) E_{\omega}\right] .
$$

In the far-field regime, we can neglect spatial modulations of both electric fields, i.e., the $\partial E_{\text {int }} / \partial Q$ and $\partial E / \partial Q$ terms. Then, relation (4) simplifies and reads

$$
\begin{aligned}
\left(\frac{\partial P}{\partial Q}\right)_{0}= & \varepsilon_{0} E_{\omega}\left[\left(\frac{\partial \chi^{(1)}}{\partial Q}\right)_{0}+2 E_{\mathrm{int}}\left(\frac{\partial \chi^{(2)}}{\partial Q}\right)_{0}\right. \\
& \left.+3 E_{\mathrm{int}}^{2}\left(\frac{\partial \chi^{(3)}}{\partial Q}\right)_{0}+\ldots\right] .
\end{aligned}
$$

The first term corresponds to spontaneous Raman scattering, whereas the second and third terms are, respectively, the linear (Pockels) and quadratic (Kerr) electro-optic vibrational contributions. As a first order approximation, we may estimate the derivatives of the susceptibilities with respect to the normal coordinate $Q$ range in the same relative scaling with the absolute susceptibilities under consideration, i.e.,

$$
\left[\frac{\partial \chi^{(n+1)}}{\partial Q}\right]_{0} /\left[\frac{\partial \chi^{(n)}}{\partial Q}\right]_{0} \approx 10^{-10} \text {. }
$$

In amorphous materials such as glasses or polymers, there is no spontaneous quadratic NLO response $\left[\chi^{(2)}\right]$ and, presumably, one would expect that the linear electro-optic vibrational contribution [second term in Eq. (5)] should be very low. Since the third order susceptibility is permitted even in isotropic materials, we should not neglect its vibrational contributions especially when the dc or optical electric fields are strong enough. Thus, for a material submitted to a strong static electric field, one may expect additional contributions to the Raman intensity arising from cross-term intensities in a first order development as

$$
\begin{aligned}
I_{\text {Raman }} \propto & \left|\left(\frac{\partial P}{\partial Q}\right)_{0}\right|^{2} \propto\left|\left(\frac{\partial \chi^{(1)}}{\partial Q}\right)_{0}\right|^{2} \\
& +\mid 4\left(\frac{\partial \chi^{(1)}}{\partial Q}\right)_{0}\left(\frac{\partial \chi^{(2)}}{\partial Q}\right)_{0} E_{\mathrm{int}} \\
& +|6|\left(\frac{\partial \chi^{(1)}}{\partial Q}\right)_{0}\left(\frac{\partial \chi^{(3)}}{\partial Q}\right)_{0} \mid \\
& \left.+\left|4\left(\frac{\partial \chi^{(2)}}{\partial Q}\right)_{0}^{2}\right|\right] E_{\mathrm{int}}^{2} \cdots .
\end{aligned}
$$

This static field dependence of the Raman polarizability has been observed in polystyrene by Aussenegg et al. ${ }^{12}$ They observed an increase of relative Raman intensity in the order of $10^{-3}-10^{-2}$ for a static field of $\sim 0.1 \times 10^{9} \mathrm{~V} / \mathrm{m}$. These experimental results range in quite good agreement with our hypotheses. Indeed, upon such a static electric field the linear $\left[2 \chi^{(2)} E_{\text {int }}\right]$ and quadratic $\left[3 \chi^{(3)} E_{\text {int }} E_{\text {int }}\right]$ electro-optic contributions could be estimated, respectively, around $2 \times 1 \%$ and $3 \times 0.01 \%$ to the total induced polarization $P$.
Let us consider now the glassy materials under investigation. The static electric field induced in poled glasses can be estimated through Eq. (1). Cardinal et al. have reported a $\chi^{(3)}$ value of $54 \times 10^{-22} \mathrm{~m}^{2} / \mathrm{V}^{2}$ for a bpn43 sample. ${ }^{13}$ As we measured a $\chi^{(2)}$ value ranging in $1-3 \mathrm{pm} / \mathrm{V}$ for this particular glass composition, ${ }^{6}$ we estimate that the internal electric field ranges in the order of $10^{9} \mathrm{~V} / \mathrm{m}$. We recall that for this kind of poled glasses, the quadratic NLO response measured by the Maker fringes technique $\left[\chi^{(2)}(-2 \omega ; \omega, \omega)\right]$ were related to the $\chi^{(3)}(-2 \omega ; 0, \omega, \omega)$ term. ${ }^{4}$ Similarly, concerning the enhanced Raman response we expect the third order term [first term of the quadratic electro-optical contribution in Eq. (7)] to be dominant versus the second order term (linear electro-optic contribution). Then, in a first approximation, if we assume no change in all susceptibilities responses in the NLO layer after the poling treatment, the relative Raman enhancement, $\Delta I_{r}$, can be expressed as follows:

$$
\Delta I_{r}=\frac{I_{p}-I_{0}}{I_{0}} \propto \frac{6 E_{\mathrm{int}}^{2}\left[\frac{\partial \chi^{(3)}}{\partial Q}\right]_{0}}{\left[\frac{\partial \chi^{(1)}}{\partial Q}\right]_{0}},
$$

where $I_{p}$ and $I_{o}$ are the Raman intensities in the NLO layer and in the bulk, respectively. With an internal field of $10^{9} \mathrm{~V} / \mathrm{m}$, Eq. (8) suggests an enhancement in the order of $6 \%$, which is more than an order of magnitude lower than the increase in Raman intensity observed in this work $(\sim 80 \%)$. It may indicate that our assumption of unchanged susceptibilities responses in the NLO layer is not valid. As a matter of fact, $\chi^{(1)}$ should be affected by the departure of $\mathrm{Na}$ ions, a process leading to the decrease of the index of refraction. ${ }^{14}$ Concerning $\chi^{(3)}$, some of us have recently evidenced that, for an unpoled glass at this composition, around $50 \%$ of the Kerr coefficient originates from vibrational contributions. ${ }^{15}$

Finally, the observed large changes in composition after thermal poling, due to the $\mathrm{Na}$ ions migration, may have induced large changes in the first and third order susceptibilities within the NLO layer. Such an effect could make the $6 E_{\text {int }}^{2}\left[\partial \chi^{(3)} / \delta Q\right]_{0}$ and $\left[\partial \chi^{(1)} / \delta Q\right]_{0}$ terms in Eq. (8) ranging in the same order and, thus, account for the observed intensity enhancement. On the other hand, if a reorientation process occurs after poling this may also induce an intrinsic $\chi^{(2)}$ that would contribute to the Raman intensity mainly through the term $4\left[\partial \chi^{(1)} / \partial Q\right]_{0}\left[\partial \chi^{(2)} / \partial Q\right]_{0} E_{\text {int }}$. Further experiments are currently scheduled to confirm these hypotheses and to get more accurate knowledge of the susceptibilities responses in the NLO layer.

\section{B. Structural evolution induced by thermal poling}

Possible structural mechanisms accounting for the observed spectral trends in Figs. 4 and 5 are now discussed. We recall that the NLO layer formed by thermal poling is characterized by a depletion of sodium ions. ${ }^{4}$ Thus, the decrease in intensity of the $900 \mathrm{~cm}^{-1}$ band characteristic of $\mathrm{NbO}_{6}$ octahedral with $\mathrm{Nb}-\mathrm{O} \cdots \mathrm{Na}^{+}$bonds is consistent with a migration of sodium ions from the anode area.

To understand the effect of poling on the bands measured at 720 and $820 \mathrm{~cm}^{-1}$, we consider analogous crystal- 
line niobate materials. The crystalline phase $\mathrm{NbPO}_{5}$ consists of chains made of corner-sharing $\mathrm{NbO}_{6}$ octahedra, with $\mathrm{PO}_{4}$ tetrahedra acting as chain cross links. This induces a distortion of $\mathrm{NbO}_{6}$ octahedra, which have four identical coplanar $\mathrm{Nb}-\mathrm{O}$ bonds $(1.97 \AA)$ and two unequal $\mathrm{Nb}-\mathrm{O}$ bonds perpendicular to the plane (1.78 and $2.32 \AA$ ). In this case, the symmetric stretching of $\mathrm{NbO}_{6}$ octahedra occurs at around $800 \mathrm{~cm}^{-1} .{ }^{16}$ Also, bands between 650 and $750 \mathrm{~cm}^{-1}$ appear in niobate oxides possessing block structures with distorted $\mathrm{NbO}_{6}$ octahedral. For example, in crystalline phases $\mathrm{H}-\mathrm{Nb}_{2} \mathrm{O}_{5}$ and $(\mathrm{P}$ or $\mathrm{V}) \mathrm{Nb}_{9} \mathrm{O}_{25}$ the corner-sharing $\mathrm{NbO}_{6}$ octahedra, which are slightly distorted with $\mathrm{Nb}-\mathrm{O}$ bonds ranging from 1.9 to $2 \AA$, exhibit their symmetric stretching in the range of $600-650 \mathrm{~cm}^{-1}$. This is in comparison to edgesharing $\mathrm{NbO}_{6}$ octahedra (highly distorted, with $\mathrm{Nb}-\mathrm{O}$ bonds ranging from 1.7 to $2.3 \AA$ ), which have the symmetric stretching mode in the range of $650-750 \mathrm{~cm}^{-1} .^{17}$

Based on the above considerations, the additional spectral changes in the NLO layer indicated by an intensity decrease at $820 \mathrm{~cm}^{-1}$ and increase at $720 \mathrm{~cm}^{-1}$ (see Fig. 5) may arise from complex structural reorganizations in the phosphoniobate glass matrix. Although no definite conclusions can be drawn at present, the observed spectral trends suggest a distortion of $\mathrm{NbO}_{6}$ octahedra induced by the thermal poling treatment. Such a spatial reorganization, triggered by the migration of sodium ions, would compensate for the excess negative charge in the NLO layer without destroying the glassy network stressed under the strong internal dc electric field.

The observed increase of the relative proportion of distorted $\mathrm{NbO}_{6}$ octahedra in the space charge layer may originate from two different mechanisms. The first one could be a structural reconstruction from corner- to edge-sharing $\mathrm{NbO}_{6}$ octahedra; this mechanism drives a partial release of oxygen atoms and an efficient anionic migration. The second mechanism may be related to a strong local charge redistribution of the niobate network under structural distortion; this would enhance the local polarizability of the medium and would be consistent with an increase of the Raman signal in the NLO space charge layer. In any case, a more detailed structural analysis would require complementary studies of poled glasses. They may include microhyper-Raman spectroscopy to track possible interactions between $E_{\text {int }}$ and the quadratic NLO susceptibility in the Raman response ${ }^{18}$ and infrared specular reflectance to obtain additional structural information of the NLO layer. ${ }^{19}$

\section{CONCLUSIONS}

Thermally poled glasses of compositions $(1-x)$ $\times\left(0.95 \mathrm{NaPO}_{3}+0.05 \mathrm{Na}_{2} \mathrm{~B}_{4} \mathrm{O}_{7}\right)+x \mathrm{Nb}_{2} \mathrm{O}_{5}, x=0.35,0.4$, and 0.45 , have been studied by probing the anode to cathode cross section using micro-Raman spectroscopy. The spectra measured within the NLO layer revealed important changes induced by poling including a clear enhancement of the Raman intensity and systematic variations in band shapes. The observed spectral variations indicate a thickness of the NLO layer in the order of 3-4 $\mu \mathrm{m}$, in very good agreement with results of previous reports. The Raman intensity enhancement has been associated to the presence of a strong static electric field induced by the migration of sodium ions away from the NLO layer. This permanent internal dc field in the NLO layer may induce additional quadratic electro-optic contribution and sequential occurrence of structural reorganizations connected, probably, to a partial migration of oxygen and, as a consequence, to a significant modification of Raman spectral density and shape.

\section{ACKNOWLEDGMENTS}

Partial support of this work by the EU (Project No. MTKD-CT-2006-042301) is gratefully acknowledged. One of the authors (V.R.) acknowledges J. L. Bruneel for technical assistance, L. Canioni for fruitful discussions, M. Couzi for careful reading of the manuscript, and financial support from Région Aquitaine.

${ }^{1}$ M. Guignard, V. Nazabal, J. Troles, F. Smektala, H. Zeghlache, Y. Quiquempois, A. Kudlinski, and G. Martinelli, Opt. Express 13, 789 (2005).

${ }^{2}$ Y. Luo, A. Frauenglass, and S. R. J. Brueck, Appl. Phys. Lett. 84, 4935 (2004).

${ }^{3}$ Y. Takahashi, K. Saitoh, T. Benino, T. Fujiwara, and T. Komatsu, J. NonCryst. Solids 345, 412 (2004).

${ }^{4}$ M. Dussauze, E. Fargin, M. Lahaye, V. Rodriguez, and F. Adamietz, Opt. Express 13, 4064 (2005).

${ }^{5}$ M. Dussauze, A. Malhako, E. Fargin, J. P. Manaud, V. Rodriguez, and F. Adamietz, J. Appl. Phys. 100, 013108 (2006).

${ }^{6}$ M. Dussauze, A. Malahko, E. Fargin, V. Rodriguez, and F. Adamietz, Opt. Mater. 28, 1417 (2006).

${ }^{7}$ T. Cardinal, E. Fargin, G. Le Flem, and S. Leboiteux, J. Non-Cryst. Solids 222, 228 (1997).

${ }^{8}$ A. Malakho, M. Dussauze, E. Fargin, B. Lazoryak, V. Rodriguez, and F. Adamietz, J. Solid State Chem. 178, 1896 (2005).

${ }^{9}$ M. Dussauze, O. Bidault, E. Fargin, M. Maglione, and V. Rodriguez, J. Appl. Phys. 100, 034905 (2006).

${ }^{10}$ S. W. Martin, Eur. J. Solid State Inorg. Chem. 28, 163 (1991); L. L. Velli, C. P. Varsamis, E. I. Kamitsos, M. Moenke, and D. Ehrt, Phys. Chem. Glasses 46, 178 (2005).

${ }^{11}$ A. A. Lipovskii, D. K. Tagantsev, A. A. Vetrov, and O. V. Yanush, Opt. Mater. 21, 749 (2003).

${ }^{12}$ F. R. Aussenberg, M. E. Lippitsch, and R. Moller, Opt. Commun. 40, 263 (1982).

${ }^{13}$ T. Cardinal, E. Fargin, G. Le Flem, and S. Leboiteux, Eur. J. Solid State Inorg. Chem. 33, 597 (1996).

${ }^{14}$ D. E. Carlson, K. W. Hang, and G. F. Stockadle, J. Am. Ceram. Soc. 57, 295 (1973).

${ }^{15}$ A. Royon et al., Phys. Rev. B (in press).

${ }^{16}$ G. T. Stranford and R. A. Condrate, J. Solid State Chem. 52, 248 (1984).

${ }^{17}$ A. A. McConnel, J. S. Anderson, and C. R. N. Rao, Spectrochim. Acta, Part A 32A, 1067 (1976).

${ }^{18}$ V. Rodriguez, D. Talaga, F. Adamietz, J. L. Bruneel, and M. Couzi, Chem. Phys. Lett. 431, 190 (2006).

${ }^{19}$ P. M. Machowski, C. P. Varsamis, and E. I. Kamitsos, J. Non-Cryst. Solids 345-346, 231 (2004) 